\title{
The Potential Use of Platelet-Rich Plasma to Reconstruct the Microtia Chondrocyte in Human Auricular Cartilage Regeneration
}

\author{
Wei-Hong Chen, ${ }^{1,2}$ Hen-Yu Liu, ${ }^{1,2}$ Ching-Yu Tsai, ${ }^{1,2}$ Chia-che Wu, ${ }^{3}$ Hong-Jian Wei, ${ }^{1,2}$ \\ Alice Liu, ${ }^{1,2}$ Ming-Tang Lai, ${ }^{3}$ Chiung-Fang Huang, ${ }^{4}$ and Win-Ping Deng ${ }^{1,2}$ \\ ${ }^{1}$ Stem Cell Research Center, Taipei Medical University, Taipei 110, Taiwan \\ ${ }^{2}$ Graduate Institute of Biomedical Materials and Tissue Engineering, Taipei Medical University, Taipei 110, Taiwan \\ ${ }^{3}$ Department of Otolaryngology, Wan-Fang Hospital, Taipei Medical University, Taipei 116, Taiwan \\ ${ }^{4}$ Department of Dentistry, Taipei Medical University Hospital, Taipei 110, Taiwan \\ Correspondence should be addressed to Chiung-Fang Huang; d642078@yahoo.com.tw \\ and Win-Ping Deng; wpdeng@ms41.hinet.net
}

Received 14 November 2014; Revised 4 March 2015; Accepted 4 March 2015

Academic Editor: Ilaria Armentano

Copyright (C) 2015 Wei-Hong Chen et al. This is an open access article distributed under the Creative Commons Attribution License, which permits unrestricted use, distribution, and reproduction in any medium, provided the original work is properly cited.

\begin{abstract}
Microtia is characterized as an incomplete auricular development and surgical reconstruction for microtia is still limited even with emerging developments. This study aimed to apply bionanomaterials (PRP/collagen scaffold) for human auricular neocartilage reconstruction by using microtia chondrocytes. The results showed that PRP (TGF- $\beta 1750 \mathrm{pg} / \mathrm{mL}$ and $1 \mathrm{ng} / \mathrm{mL}$ ) increased cell viability of microtia chondrocytes during in vitro 9-day cultures. Additionally, chondrogenic-specific mRNA of Aggrecan and type II collagen (Col II) was significantly and continuously expressed with PRP treatment during the 21-day in vitro expansion. Tissue engineering of auricular neocartilage was performed by seeding microtia chondrocytes in bionanomaterials (PRP/collagen scaffold) 3-dimensional (3D) cultures. Immunohistochemistry (IHC) of Col II showed intensive signals between cells and matrix after 4-week cultures. Conclusion. Our results demonstrated that PRP promotes proliferation and redifferentiation of microtia chondrocytes and provides regenerative potentials in auricular neocartilage reconstruction.
\end{abstract}

\section{Introduction}

Microtia, a malformed appearance of auricle, arises from incomplete skeleton development and results in structural abnormalities of auricle. The prevalence rate of microtia varies from 0.83 to 17.4 per 10,000 of birth in different population [1] and its accurate etiology is still not well understood. In addition, more than $80 \%$ of microtia patients also suffer from aural atresia [2]. Clinical correction of microtia requires specialized surgical procedures. Autologous cartilage implantation has been considered as an ideal approach to avoid immunological rejection and disease transmission. Chondrocytes from an autologous auricle have been successfully applied for auricular cartilage reconstruction in vitro and in vivo $[3,4]$. For example, auricular reconstruction using costal cartilage from a rib has been widely applied for microtia repair $[5,6]$. However, complex surgical procedures will cause multiple risks including initial postoperation pain, chest-wall deformity, implantation site scarring, and gradual resorption of the implanted costal cartilage. Hence, modified techniques are needed for advanced microtia reconstruction.

Tissue-engineered cartilage provides extra cartilaginous tissue for cosmetic surgery of the head and neck [7]. Among components for tissue engineering, specific cell type plays an important role in cartilage reconstruction. In our previous studies, human mesenchymal stem cells (MSCs), articular chondrocytes, and nucleus pulposus were employed in collagen scaffold to regenerate neocartilage [8-10]. To address auricle repair, chondrocytes isolated from normal auricle were generally used $[7,11,12]$. A tissue-engineered 
auricular cartilage was successfully created in a xenograft nude mouse model [4]. However, the source of human auricular chondrocytes from normal tissue is limited. Genetic and morphologic differences of elastic chondrocytes between normal auricle and microtia have not been clarified. Tissueengineered cartilage formation by microtia chondrocytes also showed similar elastic features of normal auricular chondrocytes [13]. Thus, cells isolated from autologous cartilage are definitely ideal for repair that also prevents immune rejection and transmittable disease. To harvest sufficient cell numbers without losing chondrogenic phenotypes is important for repair of cartilaginous defects. Moreover, microtia, obtained from patients, has limitations such as loss of cell potential with high passage number. In cell culture, the doubling time and cellular senescence of primary microtia chondrocytes can increase and be insufficient for repeated or research use.

Hence, culture medium supplemented with growth factors or cytokines has been developed. Human ear chondrocytes cultured in fibroblast growth factor 2 (FGF2) proliferated higher than serum containing medium and expressed a more differentiated phenotype [14]. The addition of TGF$\beta 1$, insulin-like growth factor (IGF), and FGF-2 was also reported to induce chondrogenic redifferentiation in scaffold reconstruction resembling native auricular cartilage $[7,15$, 16]. Recently, platelet-released growth factors comprised in platelet-rich plasma (PRP) were demonstrated to encourage wound healing in musculoskeletal injuries [17, 18]. PRP stimulated human nucleus pulposus (NP) regeneration through TGF- $\beta 1$ has been demonstrated in our previous study [10]. We have also examined the fact that PRP promotes ex vivo and in vivo NP regeneration [19]. In addition, the role of PRP combined embryonic fibroblast in the treatment of osteoporosis was also confirmed [20]. We have evaluated the efficacy of PRP on microtia chondrocytes to regenerate tissue-engineered auricular cartilage. An advanced cell/growth factor-based therapeutic strategy was developed for future head and neck surgical procedure.

\section{Materials and Methods}

2.1. Chondrocyte Isolation and Culture. The auricular cartilage fragments were harvested from microtia patients. These volunteers provided informed consent for the use of their cartilage, as required by the Ethics Committee of Taipei Medical University Municipal Wan Fang Hospital. Microtia cartilage was minced into $1 \mathrm{~mm}^{3}$ pieces and washed three times in phosphate-buffered saline (PBS). After being digested with $0.3 \%$ type I collagenase (sigma) containing penicillin (100 units/mL; GIBCO-BRL) and streptomycin $(0.1 \mathrm{mg} / \mathrm{mL}$; GIBCOBRL) for 15 hours at $37^{\circ} \mathrm{C}$, the suspended cells were pelleted and then resuspended in DMEM/F12 (Dulbecco's modified Eagle's medium/F12 Ham; GIBCO-BRL, Grand Island, NY) containing $10 \%(\mathrm{v} / \mathrm{v})$ fetal bovine serum (FBS; GIBCO-BRL) in an incubator at $37^{\circ} \mathrm{C}$ with $5 \% \mathrm{CO}_{2}$. Culture medium was changed every 3 days before subsequent experiments. In addition, we counted the cell numbers of primary microtia chondrocytes at different passage number (P1, P2, and P3) in cultures with or without PRP treatment to determine the cell proliferation.

2.2. PRP Preparation and TGF- $\beta 1$ Concentration Evaluation. PRP was extracted from total blood and activated as previously described $[10,20]$. Subsequently, the TGF- $\beta 1$ was used as indicator and quantitatively analyzed using a Quantikine enzyme-linked immunosorbent assay (ELISA) kit (\#DB100, R\&D Diagnostics, Wiesbaden, Germany) in order to confirm the consistency of PRP for in vitro utilization and to determine the most appropriate concentration for study. We prepared a dilution series of TGF- $\beta 1$ standards (\#890207) in $100 \mu \mathrm{L}$ volumes in 96-well microtiter plates coated with TGF- $\beta 1$-receptor II. Conversion of TGF- $\beta 1$ to its active form was necessary to estimate total TGF- $\beta 1$ since a large proportion of TGF- $\beta 1$ is often present in a latent form. The $0.1 \mathrm{~mL}$ PRP solution was then mixed with $0.1 \mathrm{~mL}$ $2.5 \mathrm{~N}$ acetic acid/10 M urea, incubated at room temperature for $10 \mathrm{~min}$, and neutralized by an addition of $0.1 \mathrm{~mL}$ of $2.7 \mathrm{~N}$ $\mathrm{NaOH} / 1 \mathrm{M}$ HEPES (N-[2-hydroxyethyl] piperazine-N0-[2ethanesulfonic acid]; Sigma) (\#H-7523).

2.3. MTT Assay. The analytic reagent tetrazolium salt MTT [(3-(4,5-dimethylthiazol-2-yl)-2, 5-diphenyl tetrazolium bromide), Roche, Mannheim, Germany] assay was used for determining cell viability. The microtia chondrocytes were seeded into 96 -well plate at a density of $2 \times 10^{4}$ cells $/ \mathrm{mL}$ and treated with or without PRP, while the experimental controls were cultured in $1 \%$ FBS containing DMEM/F-12. The MTT reagent was added into each well on days 1, 3, 5, 7, and 9. At the time points, OD values (OD 595-OD 690) were analyzed $4 \mathrm{~h}$ after the MTT reaction using Multiskan PC (Thermo Labsystem, Franklin, MA) and cell survival curves were then plotted against time.

2.4. Gene Expression Analysis. Expressions of type II collagen (Col II) and Aggrecan mRNA of PRP and control groups were determined by semiquantitative reverse transcription polymerase chain reaction (RT-PCR) of 7-, 14-, and 21-day monolayer cultures. Total RNA was isolated using TRIzol reagent (Invitrogen Life Technologies, Carlsbad, CA, USA) and used for first stand cDNA synthesis. RNA was reversetranscribed utilizing SuperScript III (Invitrogen Life Technologies) and an Oligo $\mathrm{d}(\mathrm{T})_{12-18}$ primer. The reaction mixture contained $10 \mathrm{mM}$ dNTP mix, 10X RT buffer, $25 \mathrm{mM} \mathrm{MgCl}$, $0.1 \mathrm{M}$ DTT, RNase inhibitor, and RNase $\mathrm{H}$ in a total volume of $20 \mu \mathrm{L}$. PCR was performed using primers for type II collagen, Aggrecan, and gyceraldehde-3-phosphate dehydrogenate $(\mathrm{GAPDH})$. The primer sequences are listed as follows: Col II-forward-5'-GAA CAT CAC CTA CCA CTG CAA G-3 ${ }^{\prime}$, reverse- $5^{\prime}$-GCA GAG TCC TAG AGT GAC TGA G-3'; Aggrecan-forward 5'-GCC TTG AGC AGT TCA CCT TC-3', reverse- $5^{\prime}$-CTC TTC TAC GGG GAC AGC AG-3'. PCR amplification in a final volume of $50 \mu \mathrm{L}$ containing $2.5 \mathrm{mM}$ dNTP, $25 \mathrm{mM} \mathrm{MgCl}_{2}$, specific primers, and Taq DNA polymerase (Invitrogen Life Technologies). The PCR was initiated for $5 \mathrm{~min}$ at $95^{\circ} \mathrm{C}$ and cycled 35 times at $94^{\circ} \mathrm{C}$ for $1 \mathrm{~min}, 72^{\circ} \mathrm{C}$ for $1 \mathrm{~min}$, and $72^{\circ} \mathrm{C}$ for $5 \mathrm{~min}$. 
2.5. Reconstruction of Tissue-Engineered Auricular Chondrocytes in Bionanomaterials and Immunohistochemical Analysis. The three-dimensional (3D) constructs were formed by bionanomaterials ( $2 \mathrm{mg} / \mathrm{mL}$ purified type I and type II collagen and mixed with/without PRP), as previously described in this laboratory $[9,10,21]$. Suspended cells were seeded at a density of $1 \times 10^{6}$ cell $/ \mathrm{mL}$ onto the collagen matrix and then cultured in an incubator at $37^{\circ} \mathrm{C}$ with $5 \% \mathrm{CO}_{2}$. Medium with/without PRP was changed every 3 days before subsequent experiments. After 2 and 4 weeks, the $3 \mathrm{D}$ constructs were then histologically analyzed. They were fixed with $10 \%$ phosphate buffered formalin for $24 \mathrm{~h}$, embedded in paraffin, and sectioned for hematoxylin and eosin (H\&E) staining. For immunohistochemical (IHC) analyses, tissue sections were stained with mouse anti-human type II collagen monoclonal antibody (Chemicon International, Temecula, CA, USA), anti-human SOX9 polyclonal antibody (Abcam, Cambridge, UK), and anti-human Aggrecan monoclonal antibody (Millipore, Darmstadt Germany).

2.6. Senescence-Associated $\beta$-Galactosidase (SA- $\beta$-Gal) Staining. SA- $\beta$ gal activity is a biomarker for developing staining in senescent cells, in which there is a lysosomal enzyme that catalyzes the hydrolysis of $\beta$-galactosides into monosaccharides only in senescent cells. All cells were fixed in the $4 \%$ formaldehyde and then the SA- $\beta$-gal assay was used to determine the cell senescence. The $\beta$-galactosidase activity at pH 6.0 produces blue perinuclear staining in senescent cells. Therefore, the blue staining in positive cells was quantitated by microscopy.

2.7. Statistical Analysis. The results of all experiments are shown as the mean \pm standard deviation (SD). Expression levels in the response to PRP were compared to $1 \%$ FBS medium and statistically evaluated using the paired $t$-test in each group.

\section{Results}

3.1. Proliferative Response of Microtia Chondrocytes Treated with PRP. Platelet-rich plasma (PRP), containing various growth factors, was extracted from total blood as described in our previous studies $[10,20]$. TGF- $\beta 1$ in PRP was recognized as the core ingredient $[22,23]$ and was used as an indicator for quantifying PRP in the following experiments. The DMEM/F12 with $1 \%$ FBS medium was designated as the basal medium and as a control group, while basal medium containing $750 \mathrm{pg} / \mathrm{mL}$ or $1 \mathrm{ng} / \mathrm{mL}$ TGF- $\beta 1$ in PRP was recognized as PRP-conditional medium. PRP-conditional medium with $750 \mathrm{pg} / \mathrm{mL}$ or $1 \mathrm{ng} / \mathrm{mL}$ TGF- $\beta 1$ showed the same timedependent increase on the proliferation of microtia chondrocytes (Figure 1(a)). Hence, PRP-conditional medium with $750 \mathrm{pg} / \mathrm{mL}$ TGF- $\beta 1$ was chosen for the following experiments. The morphologies of microtia chondrocytes treated with PRP showed a higher aggregation compared to those in control group on day 9 (Figure 1(b)).

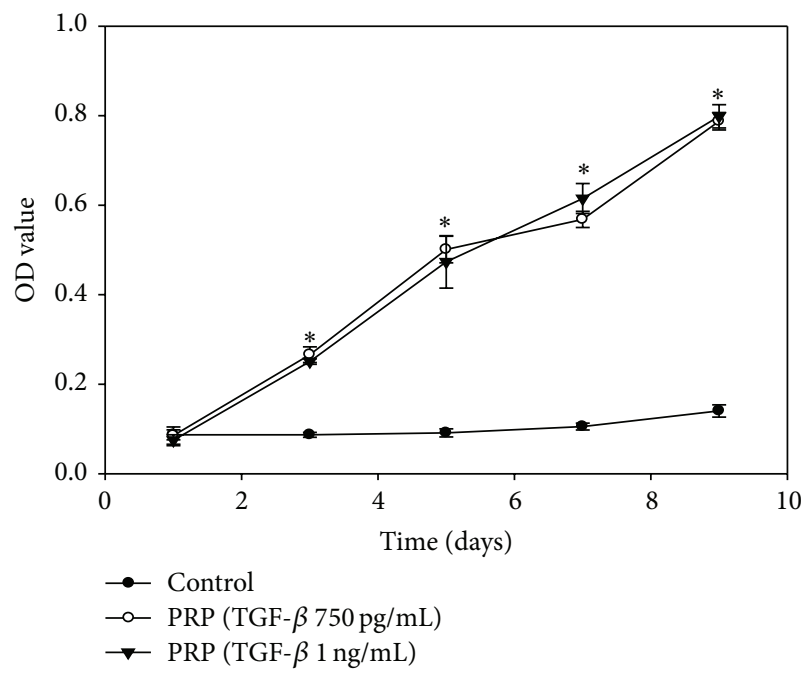

(a)

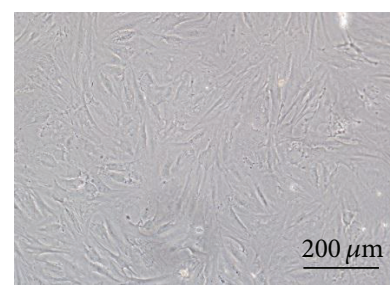

Control

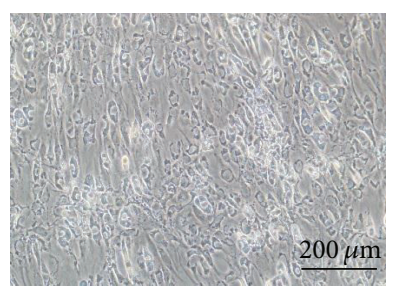

PRP (b)

FIgURE 1: Proliferative response of microtia chondrocytes treated with PRP during 9-day cultivation. (a) Proliferation of microtia chondrocytes cultured in PRP-conditional medium with $750 \mathrm{pg} / \mathrm{mL}$ TGF- $\beta 1,1000$ pg/mL TGF- $\beta 1$, and $1 \%$ FBS basal medium (control group) by MTT assay. Data was expressed as mean \pm SD $(n=3)$. (b) Morphology of microtia chondrocytes treated with and without PRP on day 9.

3.2. Aging-Related Changes in Cell Potential of Microtia Chondrocytes. We investigated the passage-dependent senescence and proliferation over multiple passages (P1, P3, and P5) of microtia chondrocytes with PRP treatment relative to the control which were cultured under normal culture conditions. Results showed significantly higher cell number in the PRP treated microtia chondrocytes in 5 passages, numbers $\mathrm{P} 1$, P3, and P5, when compared with control group (Figure 2(a)). Cellular senescence was measured by staining and quantification of senescence-associated beta-galactosidase (SA $\beta$ gal) in passage 5 cells in PRP treated and control microtia chondrocytes cultures. The percentage of SA $\beta$-gal positive cells was notably decreased in the PRP treated cells relative to the control (Figure 2(b)). Taken together, PRP treatment was very effective at increasing microtia chondrocytes in serial passage, as shown in passage numbers P1, P3, and P5 and lowering cell senescence as indicated by SA $\beta$-gal staining.

3.3. Induced Redifferentiation of Microtia Chondrocytes by $P R P$. To further determine the chondrogenic-specific gene expression of microtia chondrocyte induced by PRP, cells 


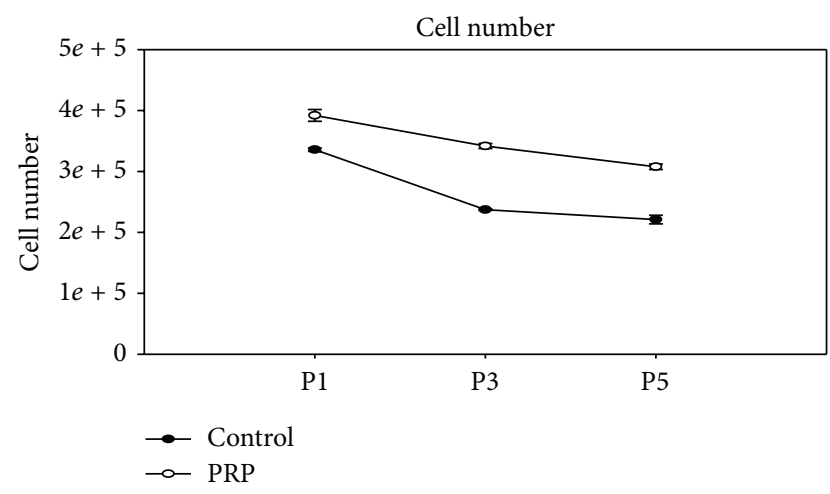

(a)

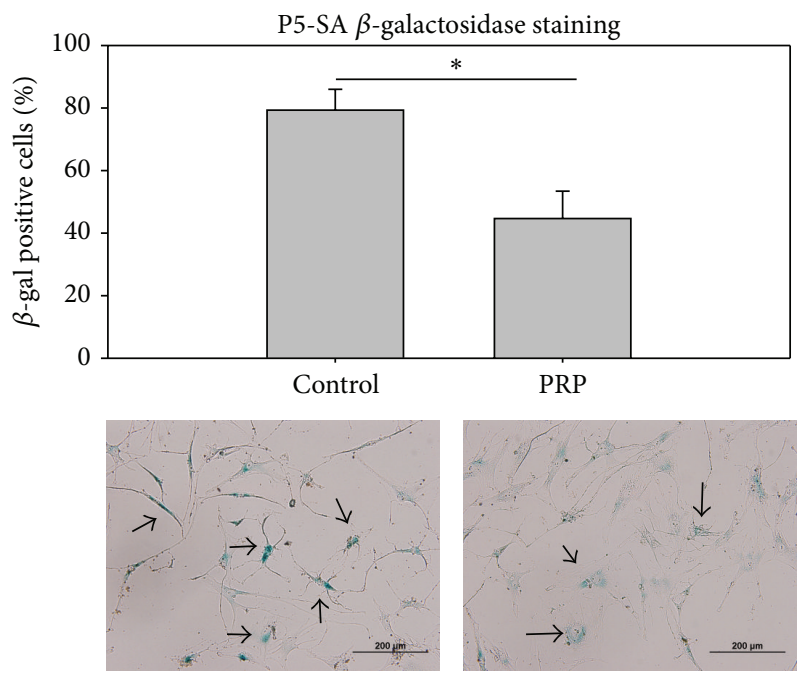

(b)

FIGURE 2: Aging effect on cell potential of microtia chondrocytes. (a) Comparative profiles of cell proliferation for microtia chondrocytes passage number in P1, P3, and P5. (b) For cellular senescence, senescence-associated beta-galactosidase activity of microtia chondrocytes during serial passage (P1, P3, and P5) in cell culture was examined in the upper panel. Quantitative representations of staining intensities were observed in the lower panel. Representative results of 3 experiments were demonstrated. ${ }^{*} P<0.05$.

were cultured in PRP-conditional medium (containing $750 \mathrm{pg} / \mathrm{mL}$ TGF- $\beta 1$ ) or basal medium. Expressions of chondrogenic genes such as Aggrecan and type II collagen (Col II) were diminished during 21-day in vitro expansion in basal medium (without PRP). However, specific mRNA in both Aggrecan and Col II was significantly and continuously expressed on day 21 in PRP treatment group (Figure 3(a)). Quantitatively, an approximately 2-fold increase in Aggrecan and 3-fold increase in Col II were observed in PRP treatment group when compared to control group (Figure 3(b)). These results indicated that chondrogenic redifferentiation of microtia chondrocytes can be induced by PRP.
3.4. Three-Dimensional Culture of Auricular Neocartilage for Microtia Chondrocytes with Bionanomaterials. To reconstruct tissue-engineered auricular cartilage, three-dimensional (3D) cultures were performed by seeding microtia chondrocytes in bionanomaterials (collagen scaffold mixed with or without PRP) and then chondrogenic-specific matrices were histologically determined (Figure 4). From gross morphology, there were no significant differences between tissue-engineered microtia cartilages cultured with and without PRP on days 14 and 28 (Figures 4(a)-4(d)). Hematoxylin and eosin (H\&E) staining showed that chondrocyte-like rounded cells of auricle in $3 \mathrm{D}$ culture and a higher cell density were observed in microtia chondrocytes cultured with PRP (Figures 4(f) and 4(h)), compared to control group on day 28 (Figure 4(g)). Immunohistochemical (IHC) staining demonstrated that staining signal for type II collagen, SOX9, and Aggrecan synthesis was more intensive and extensive in microtia chondrocytes cultured with PRP (Figures 4(j), 4(l), $4(\mathrm{n}), 4(\mathrm{p}), 4(\mathrm{r})$, and $4(\mathrm{t}))$, compared to their control groups (Figures 4(i), 4(k), 4(m), 4(o), 4(q), and 4(s)). The results indicate that PRP promotes chondrogenic differentiation of microtia in 3D cultures, supported by their mRNA expression of monolayer cultures.

\section{Discussion}

We have previously demonstrated that platelet-rich plasma (PRP) promoted chondrogenic redifferentiation in intervertebral disc (IVD) and bone regeneration $[10,19,20]$. In this study, we showed the role of PRP in auricle regeneration by using microtia chondrocytes. For tissue reconstruction, cells numbers need to be multiplied to obtain the required amount. However, it is challenging to prevent dedifferentiation during in vitro cell expansion. We and others have previously demonstrated that PRP could be recognized as a mitogen for human nucleus pulposus, stromal stem cells, osteoblasticlike cells, and fibroblast proliferation [10, 24-26]. We first demonstrated the important role of transforming growth factor- $\beta 1$ (TGF- $\beta 1$ ) in PRP and assigned TGF- $\beta 1$ as a quantity indicator for PRP concentration [10]. This is essential and accurate to determine the dosage of PRP for controlling both proliferation and redifferentiation in cartilage regeneration. TGF- $\beta 1$-induced specific $\mathrm{pSMAD} 2 / 3$ pathway was activated by PRP [10]. Similar to our previous study [10, 19, 20], PRP increased cell viability of microtia chondrocyte by 10 -fold at TGF- $\beta 1750 \mathrm{pg} / \mathrm{mL}$ and $1 \mathrm{ng} / \mathrm{mL}$, compared with $1 \%$ FBS control (Figure 1). Microtia chondrocytes also showed an aggregated morphology in the presence of PRP, indicating the upregulated chondrogenic redifferentiation phenotypes followed by abundant cell-to-cell contact [27]. Therefore, our result also showed that PRP could recover proliferation and replicative potential during cellular senescence (Figure 2).

Chondrogenic-specific genes were subsequently examined for chondrogenic redifferentiation potentials of microtia chondrocytes in the presence of PRP. From RT-PCR results, specific marker gene expression such as Aggrecan and type II collagen (Col II) was diminished during in vitro culture. It has been reported as a particular feature referring to 

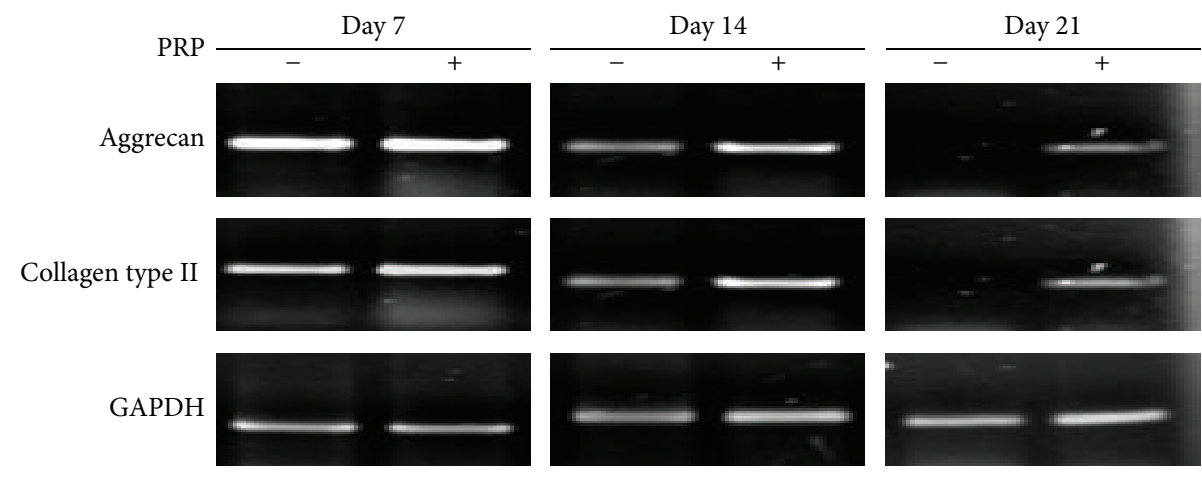

(a)
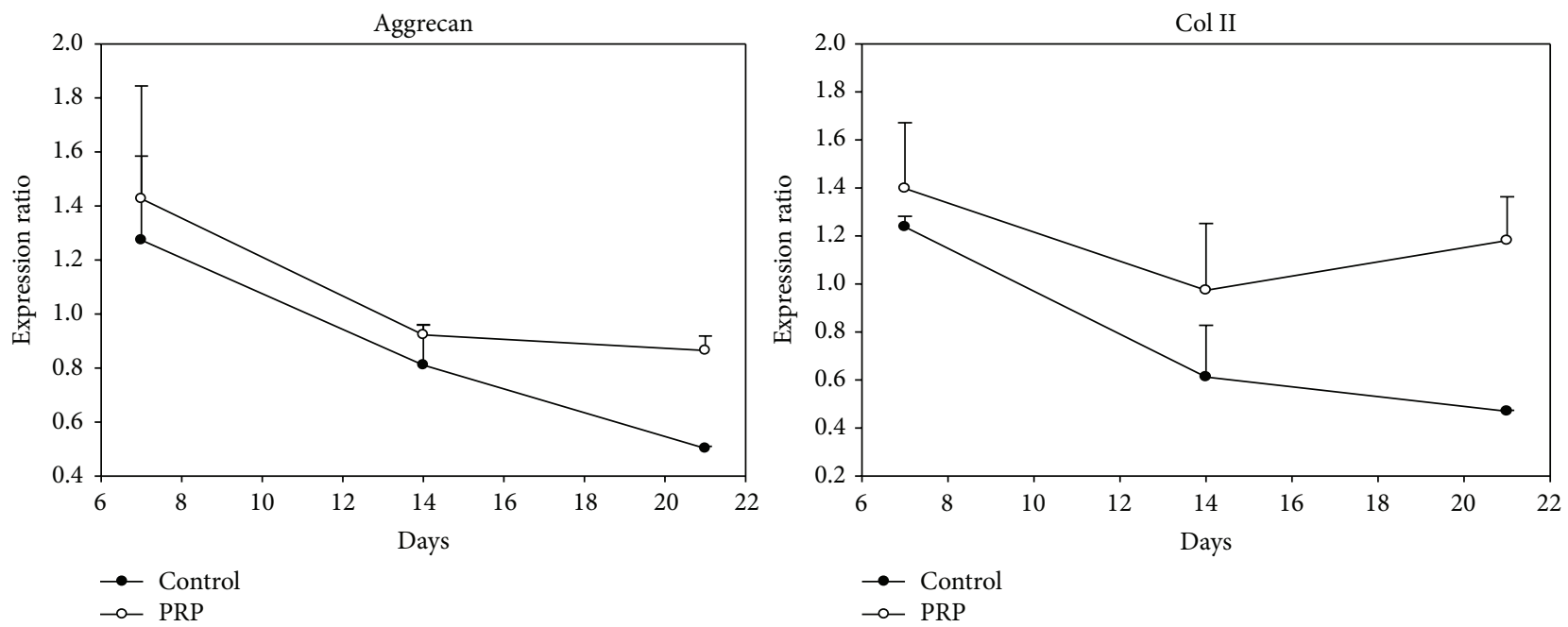

(b)

FIgURE 3: Chondrogenic-specific mRNA of microtia chondrocytes by semiquantitative RT-PCR. (a) Microtia chondrocytes were cultured with (+) and without (-) PRP during 21 days. Chondrogenic-specific mRNA including Aggrecan and type II collagen was examined, and (b) their expression ratio was normalized with respective glyceraldehydes phosphate dehydrogenase (GAPDH). ${ }^{*} P<0.05$ as PRP group compared to $1 \%$ FBS control group at the same time point, using paired $t$-test.

dedifferentiation of primary chondrocytes during expansion [28]. In Figure 3, specific mRNA of Aggrecan and Col II was significantly and continuously expressed up to 21 days compared with the normal control $(P<0.05)$. van Osch et al. reported that type II collagen in human and rabbit auricular chondrocytes was increased by TGF- $\beta 1$ and insulinlike growth factor (IGF-I) [7]. Basic fibroblast growth factor (bFGF) was also demonstrated to promote in vitro and in vivo growth of human pediatric auricular cartilage [3]. Moreover, human ear chondrocytes expanded in medium containing TGF- $\beta 1$, fibroblast growth factor-2 (FGF-2), and plateletderived growth factor bb (PDGF-bb) displayed a superior postexpansion chondrogenic potential [29]. Interestingly, all these growth factors were the core constituents of PRP. Taken together, PRP stimulates microtia chondrocyte proliferation without loss of chondrogenic-specific markers in the microtia chondrocyte expansion cultures.
For further clinical applications of auricle surgical reconstruction, PRP could be viewed as a good natural source of growth factor cocktails. In addition, the cell adhesion protein including fibrin, fibronectin, and vitronectin comprised in PRP also provides 3-dimensional (3D) architecture for fixing seeded chondrocytes [30]. Previously, we have seeded chondrocytes into collagen scaffold to reconstitute human engineered nucleus pulposus tissue and human articular cartilage [8-10]. PRP was subsequently employed in the auricular reconstruction system containing microtia chondrocyte/collagen scaffold. In Figure 4, higher cell density was only shown in 4-week PRP treated groups. Numerous lacunae surrounded cells were also observed by H\&E staining. Intensive signals of type II collagen, SOX9, and Aggrecan staining by immunohistochemistry also indicated that PRP stimulates the major chondrogenic matrix synthesized by microtia chondrocytes in 3D environment (Figure 4(l)). Both PRP 


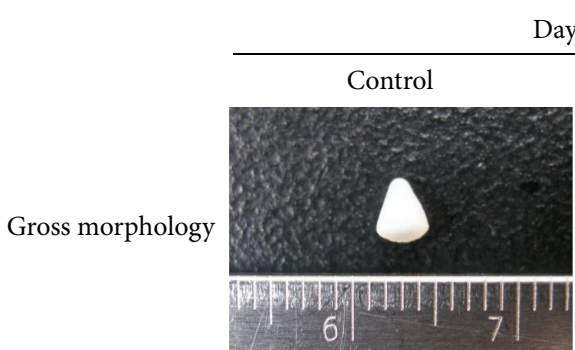

(a)

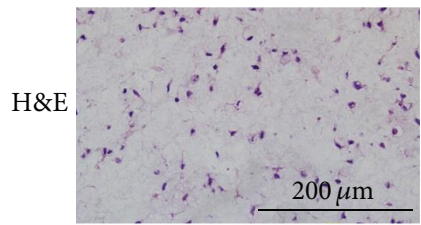

(e)

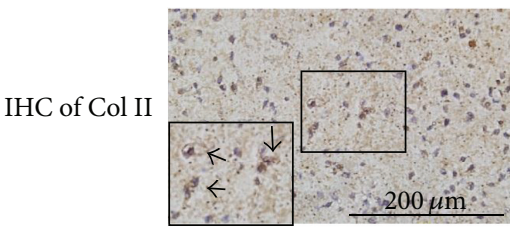

(i)

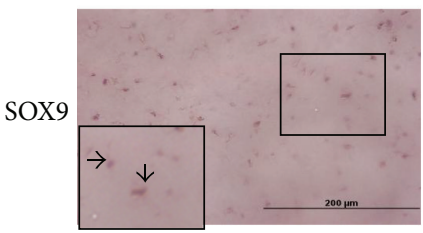

(m)

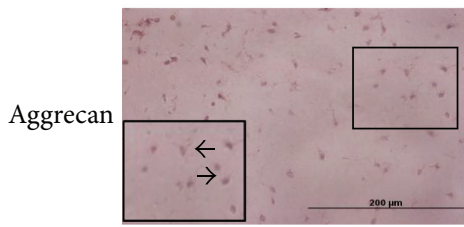

(q) ay 14

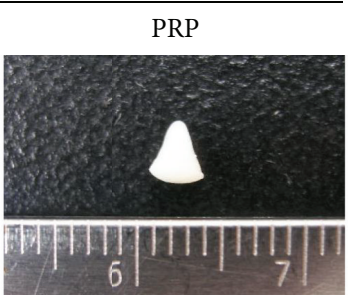

(b)

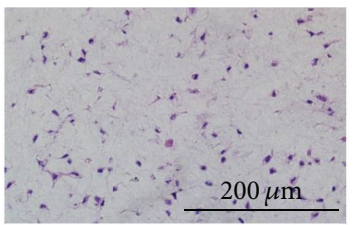

(f)

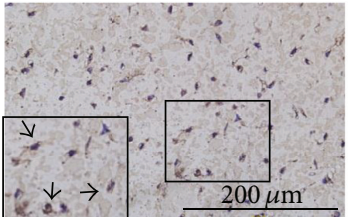

(j)

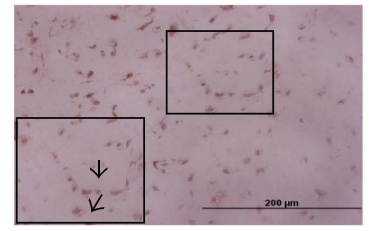

(n)

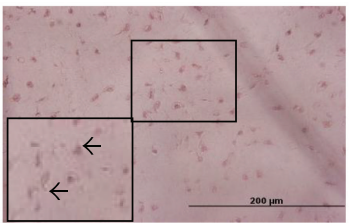

(r)

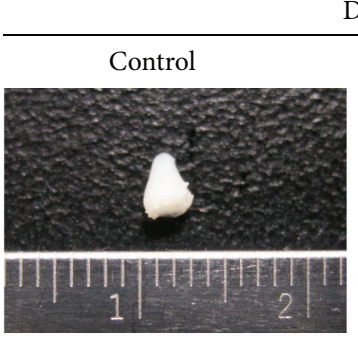

(c)

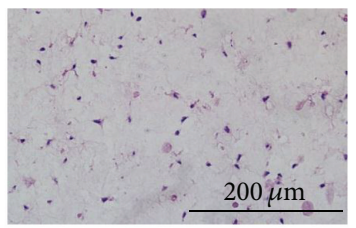

(g)

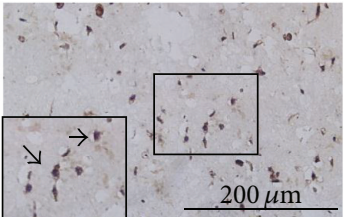

(k)

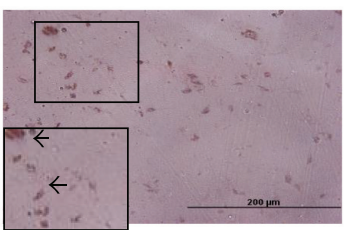

(o)

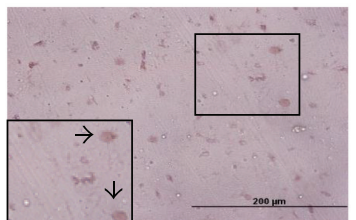

(s)
Day 28

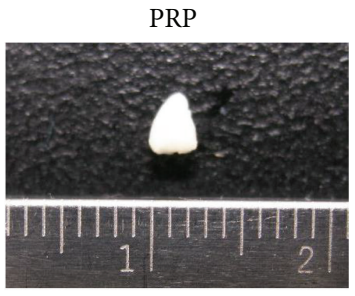

(d)

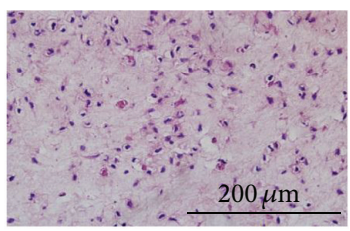

(h)

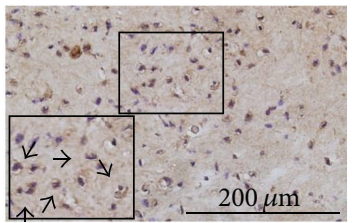

(1)

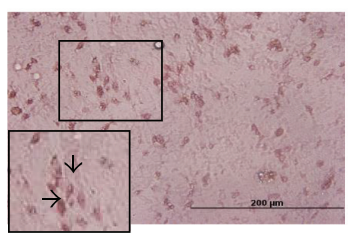

(p)

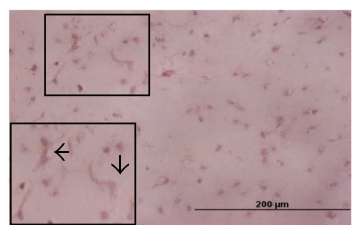

(t)

FIGURE 4: Engineered auricle tissue by microtia chondrocytes reconstructed in 3D collagen scaffold with and without PRP. Neocartilage was analyzed by their gross morphology ((a)-(d)), H\&E stain ((e)-(h)) (200x), and IHC of Col II ((i)-(l)), SOX9 ((m)-(p)), and Aggrecan stain $((\mathrm{q})-(\mathrm{t}))(200 \mathrm{x} \rightarrow 400 \mathrm{x})$ on days 14 and 28.

and collagen framework rendered essential compositions for auricular neocartilage formation.

\section{Conclusion}

In this study, we demonstrated that PRP increased the potential of microtia chondrocytes to effectively regenerate auricle tissue. Our findings not only provide a more advanced application but also contribute to a new cell/growth factor-based therapeutic strategy for clinical microtia reconstruction.

\section{Conflict of Interests}

Wei-Hong Chen, Hen-Yu Liu, Ching-Yu Tsai, Chia-che Wu, Hong-Jian Wei, Alice Liu, Ming-Tang Lai, Chiung-Fang
Huang, and Win-Ping Deng declare that they have no conflict of interests with the mentioned trademarks or companies.

\section{Authors' Contribution}

Wei-Hong Chen and Hen-Yu Liu contributed equally to this work. Chiung-Fang Huang and Win-Ping Deng contributed equally to this work.

\section{References}

[1] S. Suutarla, J. Rautio, A. Ritvanen, S. Ala-Mello, J. Jero, and T. Klockars, "Microtia in Finland: comparison of characteristics in different populations," International Journal of Pediatric Otorhinolaryngology, vol. 71, no. 8, pp. 1211-1217, 2007. 
[2] F. Alasti and G. van Camp, "Genetics of microtia and associated syndromes," Journal of Medical Genetics, vol. 46, no. 6, pp. 361369, 2009.

[3] C. A. Arévalo-Silva, Y. Cao, M. Vacanti, Y. Weng, C. A. Vacanti, and R. D. Eavey, "Influence of growth factors on tissue-engineered pediatric elastic cartilage," Archives of OtolaryngologyHead and Neck Surgery, vol. 126, no. 10, pp. 1234-1238, 2000.

[4] Y. Cao, J. P. Vacanti, K. T. Paige, J. Upton, and C. A. Vacanti, "Transplantation of chondrocytes utilizing a polymer-cell construct to produce tissue-engineered cartilage in the shape of a human ear," Plastic and Reconstructive Surgery, vol. 100, no. 2, pp. 297-304, 1997.

[5] D. E. Johns and K. A. Athanasiou, "Growth factor effects on costal chondrocytes for tissue engineering fibrocartilage," Cell and Tissue Research, vol. 333, no. 3, pp. 439-447, 2008.

[6] V. Kizkner and A. Barak, "Framework changes using costal cartilage for microtia reconstruction," Archives of OtolaryngologyHead and Neck Surgery, vol. 134, no. 7, pp. 768-770, 2008.

[7] G. J. V. M. van Osch, S. W. van der Veen, and H. L. VerwoerdVerhoef, "In vitro redifferentiation of culture-expanded rabbit and human auricular chondrocytes for cartilage reconstruction," Plastic and Reconstructive Surgery, vol. 107, no. 2, pp. 433440, 2001.

[8] W.-H. Chen, M.-T. Lai, A. T. H. Wu et al., "In vitro stagespecific chondrogenesis of mesenchymal stem cells committed to chondrocytes," Arthritis and Rheumatism, vol. 60, no. 2, pp. 450-459, 2009.

[9] W.-H. Chen, W.-F. Lai, W.-P. Deng et al., "Tissue engineered cartilage using human articular chondrocytes immortalized by HPV-16 E6 and E7 genes," Journal of Biomedical Materials Research Part A, vol. 76, no. 3, pp. 512-520, 2006.

[10] W.-H. Chen, W.-C. Lo, J.-J. Lee et al., "Tissue-engineered intervertebral disc and chondrogenesis using human nucleus pulposus regulated through TGF- $\beta 1$ in platelet-rich plasma," Journal of Cellular Physiology, vol. 209, no. 3, pp. 744-754, 2006.

[11] T. de Chalain, J. H. Phillips, and A. Hinek, "Bioengineering of elastic cartilage with aggregated porcine and human auricular chondrocytes and hydrogels containing alginate, collagen, and $\kappa$-elastin," Journal of Biomedical Materials Research, vol. 44, no. 3, pp. 280-288, 1999.

[12] H. Yamaoka, H. Asato, T. Ogasawara et al., "Cartilage tissue engineering using human auricular chondrocytes embedded in different hydrogel materials," Journal of Biomedical Materials Research Part A, vol. 78, no. 1, pp. 1-11, 2006.

[13] S. H. Kamil, M. P. Vacanti, C. A. Vacanti, and R. D. Eavey, "Microtia chondrocytes as a donor source for tissue-engineered cartilage," Laryngoscope, vol. 114, no. 12, pp. 2187-2190, 2004.

[14] E. W. Mandl, H. Jahr, J. L. M. Koevoet et al., "Fibroblast growth factor- 2 in serum-free medium is a potent mitogen and reduces dedifferentiation of human ear chondrocytes in monolayer culture," Matrix Biology, vol. 23, no. 4, pp. 231-241, 2004.

[15] P. B. Saadeh, B. Brent, B. J. Mehrara et al., "Human cartilage engineering: chondrocyte extraction, proliferation, and characterization for construct development," Annals of Plastic Surgery, vol. 42, no. 5, pp. 509-513, 1999.

[16] C. A. Arévalo-Silva, Y. Cao, Y. Weng et al., "The effect of fibroblast growth factor and transforming growth factor-beta on porcine chondrocytes and tissue-engineered autologous elastic cartilage," Tissue Engineering, vol. 7, no. 1, pp. 81-88, 2001.

[17] S. Mehta and J. T. Watson, "Platelet rich concentrate: basic science and current clinical applications," Journal of Orthopaedic Trauma, vol. 22, no. 6, pp. 433-438, 2008.
[18] S. Sampson, M. Gerhardt, and B. Mandelbaum, "Platelet rich plasma injection grafts for musculoskeletal injuries: a review," Current Reviews in Musculoskeletal Medicine, vol. 1, no. 3-4, pp. 165-174, 2008.

[19] W. H. Chen, H. Y. Liu, W. C. Lo et al., "Intervertebral disc regeneration in an ex vivo culture system using mesenchymal stem cells and platelet-rich plasma," Biomaterials, vol. 30, no. 29, pp. 5523-5533, 2009.

[20] W.-C. Lo, J.-F. Chiou, J. G. Gelovani et al., “Transplantation of embryonic fibroblasts treated with platelet-rich plasma induces osteogenesis in SAMP8 mice monitored by molecular imaging," Journal of Nuclear Medicine, vol. 50, no. 5, pp. 765-773, 2009.

[21] W.-F. Lai, J.-R. Tang, and C.-T. Chen, "Fibrication of a cartilage implant," US Patent 20030152556 A1, 2003.

[22] R. Landesberg, M. Roy, and R. S. Glickman, "Quantification of growth factor levels using a simplified method of plateletrich plasma gel preparation," Journal of Oral and Maxillofacial Surgery, vol. 58, no. 3, pp. 297-300, 2000.

[23] G. Weibrich, W. K. G. Kleis, G. Hafner, and W. E. Hitzler, "Growth factor levels in platelet-rich plasma and correlations with donor age, sex, and platelet count," Journal of Cranio-Maxillofacial Surgery, vol. 30, no. 2, pp. 97-102, 2002.

[24] P. Arpornmaeklong, M. Kochel, R. Depprich, N. R. Kübler, and K. K. Würzler, "Influence of platelet-rich plasma (PRP) on osteogenic differentiation of rat bone marrow stromal cells. An in vitro study," International Journal of Oral and Maxillofacial Surgery, vol. 33, no. 1, pp. 60-70, 2004.

[25] T. Kawase, K. Okuda, L. F. Wolff, and H. Yoshie, "Plateletrich plasma-derived fibrin clot formation stimulates collagen synthesis in periodontal ligament and osteoblastic cells in vitro," Journal of Periodontology, vol. 74, no. 6, pp. 858-864, 2003.

[26] Y. Liu, A. Kalén, O. Risto, and O. Wahlström, "Fibroblast proliferation due to exposure to a platelet concentrate in vitro is $\mathrm{pH}$ dependent," Wound Repair and Regeneration, vol. 10, no. 5, pp. 336-340, 2002.

[27] S. T. Yoon, K. S. Kim, J. Li et al., "The effect of bone morphogenetic protein-2 on rat intervertebral disc cells in vitro," Spine, vol. 28, no. 16, pp. 1773-1780, 2003.

[28] K. von der Mark, V. Gauss, H. von der Mark, and P. Mueller, "Relationship between cell shape and type of collagen synthesised as chondrocytes lose their cartilage phenotype in culture," Nature, vol. 267, no. 5611, pp. 531-532, 1977.

[29] A. G. Tay, J. Farhadi, R. Suetterlin, G. Pierer, M. Heberer, and I. Martin, "Cell yield, proliferation, and postexpansion differentiation capacity of human ear, nasal, and rib chondrocytes," Tissue Engineering, vol. 10, no. 5-6, pp. 762-770, 2004.

[30] R. E. Marx, "Platelet-rich plasma: evidence to support its use," Journal of Oral and Maxillofacial Surgery, vol. 62, no. 4, pp. 489496, 2004. 

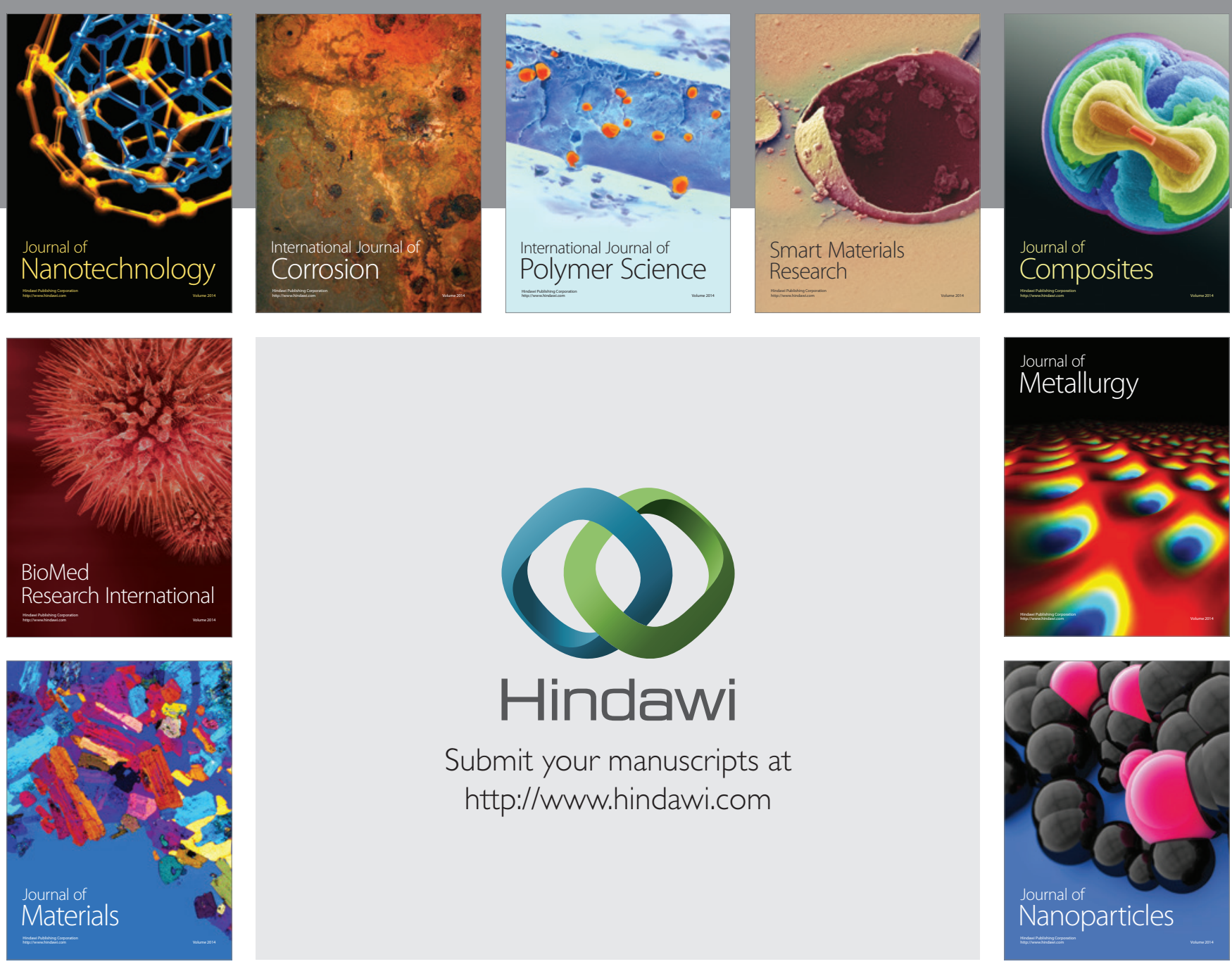

Submit your manuscripts at http://www.hindawi.com
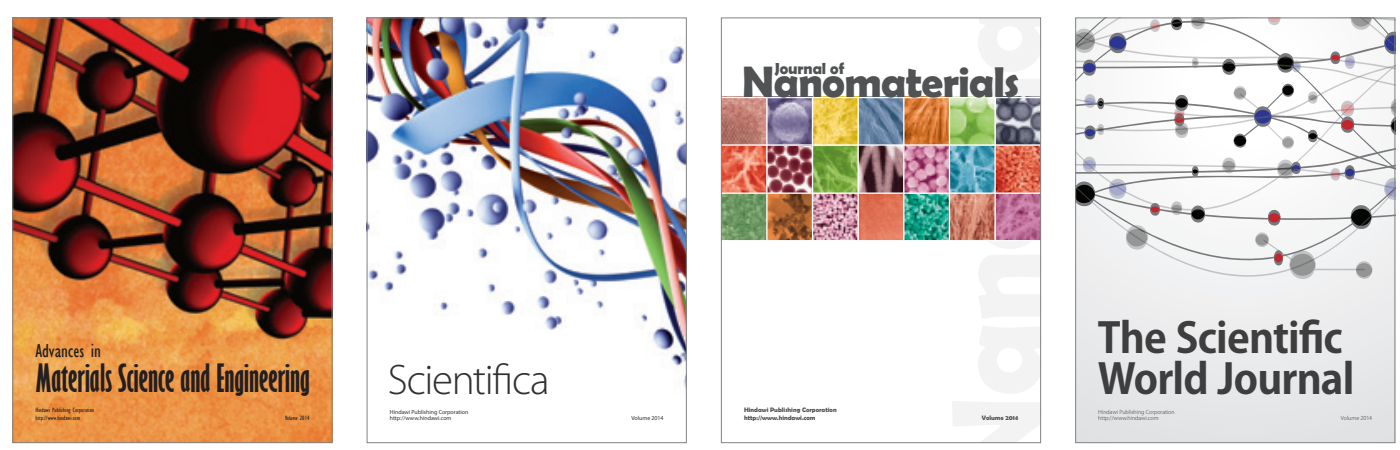

\section{The Scientific World Journal}
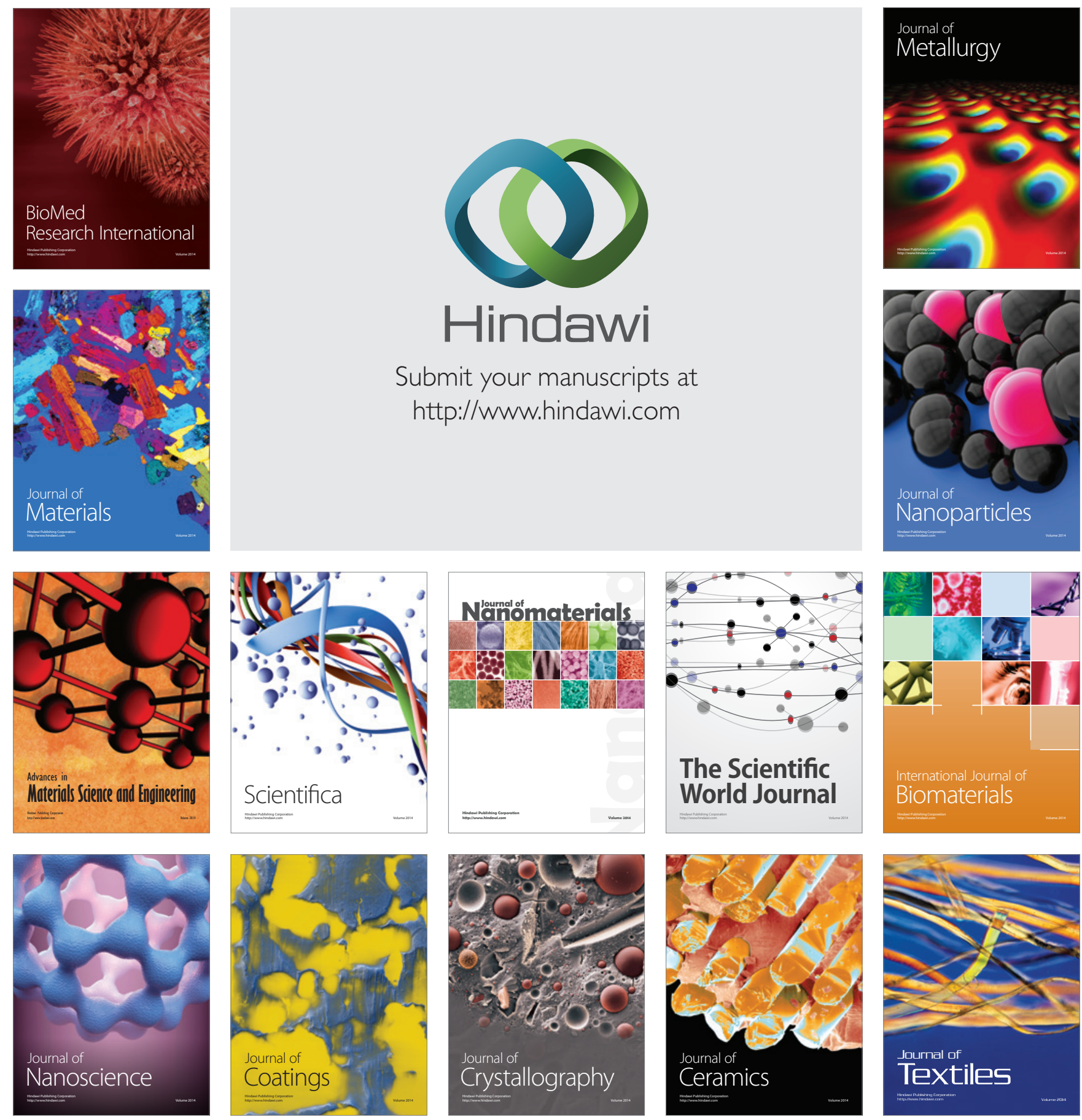